\title{
Optical coherence tomography angiography findings of choroidal neovascularization in pseudoxanthoma elasticum
}

\author{
Orly Gal-Or ${ }^{1,2,5}$, Chandrakumar Balaratnasingam ${ }^{1,2,3}$ and K Bailey Freund 1,2,4*
}

\begin{abstract}
Angioid streaks (AS) are the most common ocular manifestation in patients with pseudoxanthoma elasticum (PXE). The major cause of severe visual loss in patients with AS is choroidal neovascularization (CNV). We report the optical coherence tomography angiography (OCTA) findings of CNV in a patient with PXE and angioid streaks. A 51-year-old man with PXE presented with visual disturbance in his right eye. Best corrected visual acuity was 20/30 OD and 20/30 OS. Funduscopic examination revealed angioid streaks and type $1 \mathrm{NV}$ in his right eye. Multimodal imaging including OCTA demonstrated CNV nasal to the fovea. The morphology and configuration of CNV followed the path of the AS. OCTA combined with other multimodal imaging modalities may be a useful tool for diagnosing CNV secondary to angioid streaks in patients with pseudoxanthoma elasticum. The configuration of CNV in these may follow the path of angioid streaks implicating Bruch's membrane disruption as an important anatomical change in the pathogenesis of CNV.
\end{abstract}

Keywords: Angioid streaks, Bruch's membrane, Choroidal neovasculariztion, Multimodal imaging, Optical coherence tomography angiography

\section{Background}

Pseudoxanthoma elasticum (PXE) is a rare inherited multisystem disorder. There are many ocular manifestations associated with PXE of which angioid streaks (AS) are the most common. AS represent breaks within calcified and thickened elastic fibers of Bruch's membrane. They often do not affect visual function, but they may be complicated by the development of choroidal neovascularization $(\mathrm{CNV})$, which is a major cause of visual loss in these patients. Optical coherence tomography angiography (OCTA) is relatively new non-invasive imaging modality that demonstrates flow-characteristics of the vascular network. In this report we demonstrate the morphology

\footnotetext{
*Correspondence: kbfnyf@aol.com

${ }^{2}$ Vitreous-Retina-Macula Consultants of New York, 460 Park Avenue,

5th Floor, New York, NY 10022, USA

Full list of author information is available at the end of the article
}

and spatial relationships of $\mathrm{CNV}$ and angioid streaks using OCTA.

\section{Case presentation}

Pseudoxanthoma elasticum is an inherited multisystem disorder with a prevalence range between 1:25,000 and 1:100,000 [1]. There are many ocular manifestations associated with PXE of which angioid streaks (AS) are the most common. Angioid streaks are identified in approximately $85 \%$ of eyes with PXE and represent breaks within calcified and thickened elastic fibers of Bruch's membrane [2,3]. AS often do not affect visual function, even if they cross into the foveal area, but they may be complicated by the development of choroidal neovascularization (CNV) [4]. The occurrence of CNV is a major cause of visual loss in PXE and our knowledge concerning the pathogenesis, morphology and natural course of this complication remains limited [5]. 
Optical coherence tomography angiography (OCTA) is relatively new imaging modality that exploits flowcharacteristics within the regional circulation to construct non-invasive images of the vascular network [6]. En face images generated by OCTA also allows us to study the spatial relationships between vasculature and adjacent retinal/choroidal layers with greater precision than dye angiography. In this report, we integrate OCTA with other multimodal imaging findings to improve our understanding of CNV in the setting of angioid streaks.
The study was approved by the Western Institutional Review Board and written informed consent was provided by the patient. A 51-year-old male patient had received care for the ocular complications of pseudoxanthoma elasticum for 9 years. Best corrected visual acuity was 20/30 OD and 20/30 OS. On presentation, his right eye demonstrated AS on multimodal imaging (Fig. 1). Color fundus and red-free photographs revealed the AS in the peripapillary area radiating to the periphery of the posterior pole and towards the fovea with slight foveal mottling. Fluorescein angiography showed variable
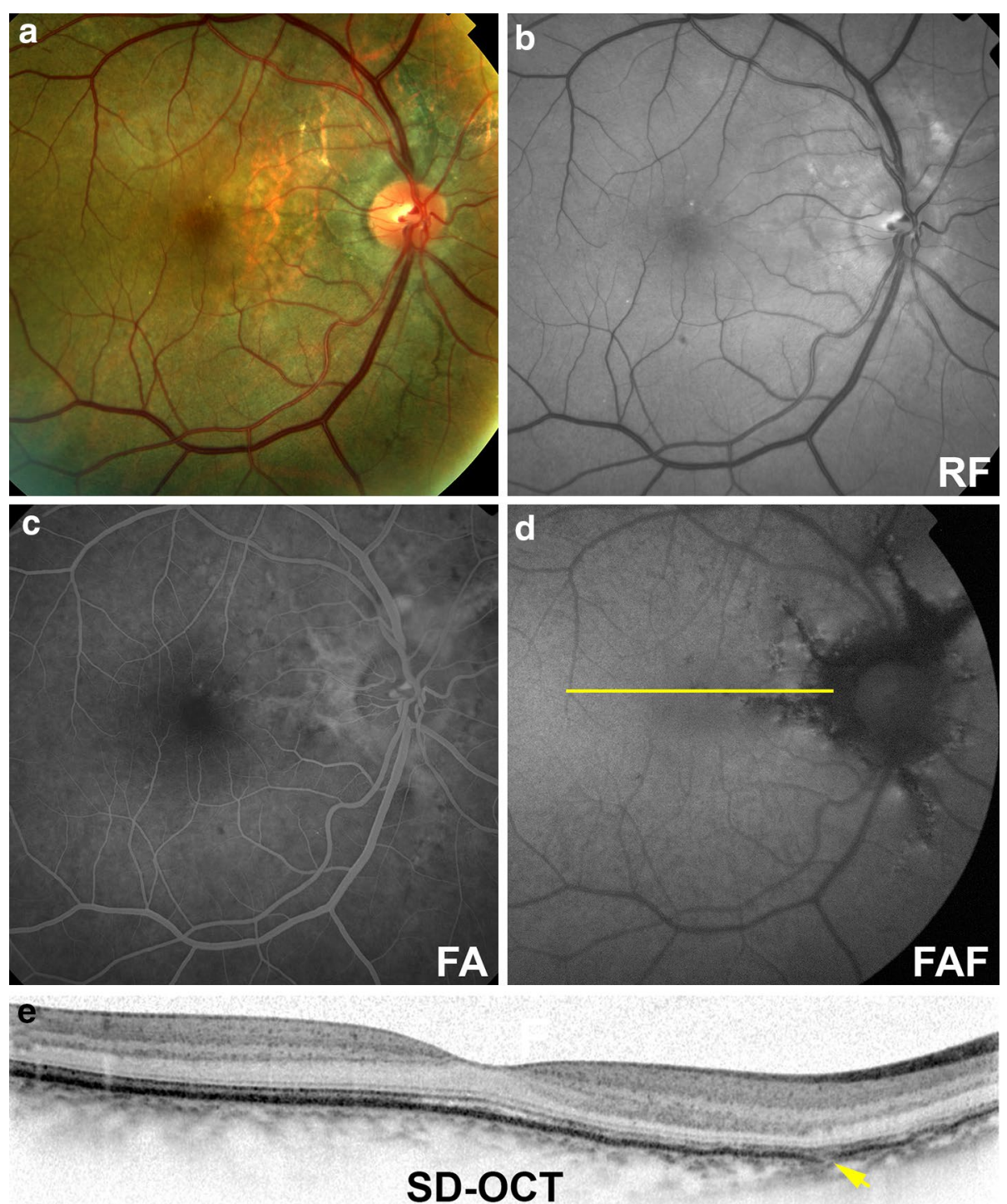

Fig. 1 Multimodal imaging findings of angioid streaks at baseline visit. Color (a) and red-free (RF) photographs (b) of the right eye show angioid streaks at the peripapillary region radiating into the fovea. Late-phase fluorescein angiogram ( $\mathbf{c}$; FA) shows variable staining of the angioid streaks with no leakage. Fundus autofluorescence (d; FAF) imaging shows areas of hypoautofluorescence in the peripapillary area that are greater in size than the corresponding AS seen on color photography. AS correlate to breaks within the Bruch's membrane (yellow arrow) as seen on spectral domain optical coherence tomography (e; SD-OCT). The yellow line indicates the location of the SD-OCT scan. 
staining of the AS without leakage in any phase. Fundus autofluorescence showed distinct areas of hypoautofluorescence corresponding to the AS with focal spots of increased autofluorescence alongside the AS. SD-OCT showed breaks in Bruch's membrane (BrM) with preservation of the overlying RPE. His left eye showed subfoveal CNV that was treated with photodynamic therapy (PDT) and several anti-vascular endothelial growth
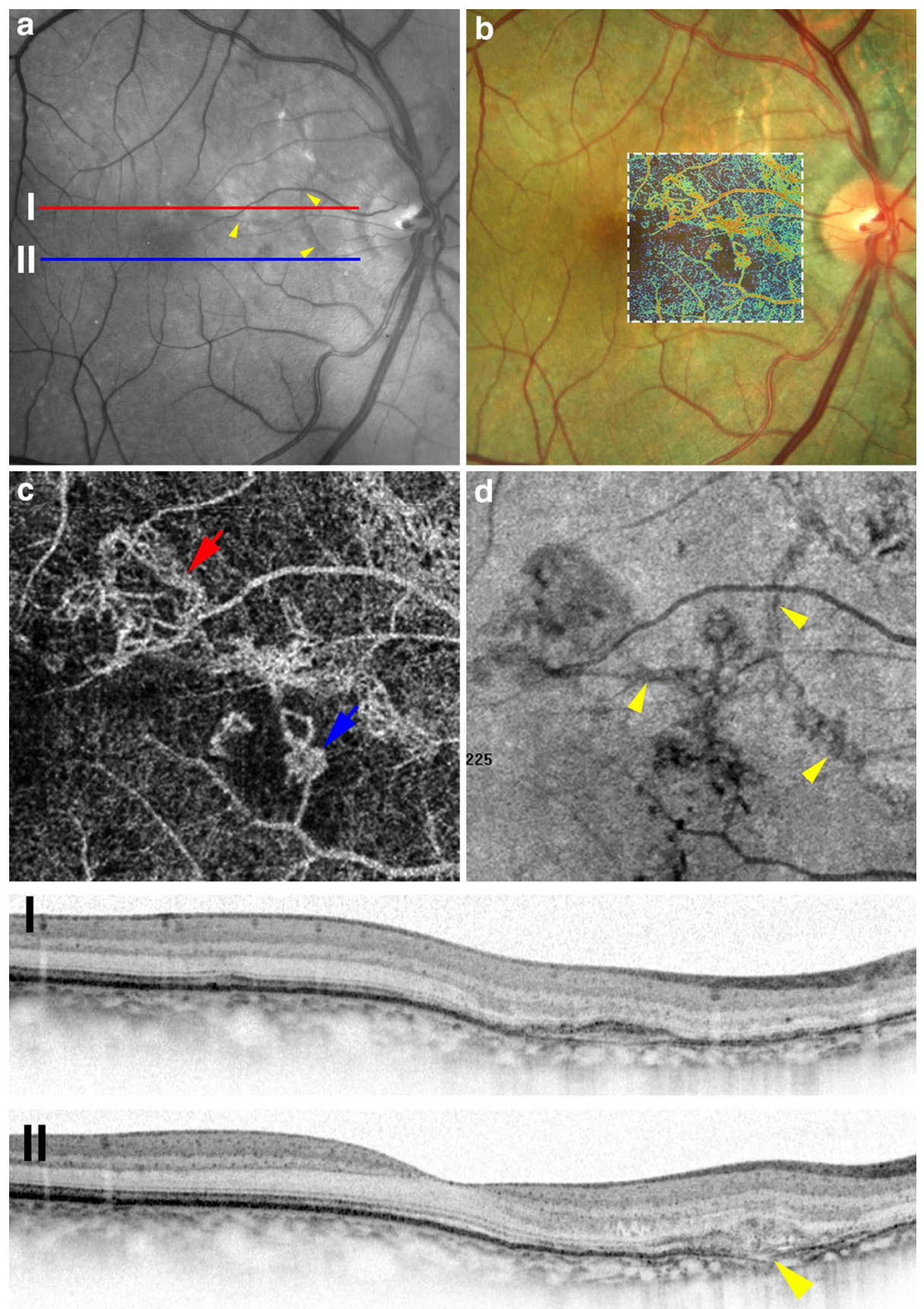

Fig. 2 Multimodal imaging findings of choroidal neovascularization. Angioid streaks are observed on the red-free image (a); however, no hemorrhage is evident. Overlay of false-colored optical coherence tomography angiography (OCTA) over the color photograph (b) outlines the area of choroidal neovascularization (CNV). En Face OCTA (c) demonstrates two area of CNV (red and blue arrows) that demonstrate a tangled morphology of vascular networks. Sites of CNV closely correlate to sites of angioid streaks (d; arrowheads) when OCTA is compared to en face reflectance images. Structural OCT scans (I and II) confirm a mixed type 1 (I) and type 2 (II) neovascular lesion which arise in proximity to sites of Bruch's membrane disruption. 
factor (anti-VEGF) injections with eventual subretinal fibrosis. Final best corrected visual acuity in the left eye was 20/30.

Five years later, the patient presented with visual disturbances involving his right eye. Dilated funduscopic examination revealed juxtafoveal CNV. The patient underwent monthly injections of bevacizumab $(1.25 \mathrm{mg} / 0.05 \mathrm{ml})$ for 11 months that achieved regression of the lesion.

Two years following this, there was recurrence of $\mathrm{CNV}$ in the same eye. Multimodal imaging characteristics of this CNV are provided in Figs. 2 and 3. There was no evolution in the size of the angioid streaks since presentation. OCTA demonstrated CNV nasal to the fovea. The morphology of CNV was characterized by a tangled network of vessels [7]. The configuration of CNV closely followed the trajectory of AS. Structural OCT revealed type 1(between RPE and BrM) and type 2 (sub-retinal) neovascularization. Breaks in BrM were seen on the outer aspect of the area of neovascularization. Visualization of the ultrastructural features of $\mathrm{CNV}$ using indocyanine green angiography was limited in this case due to the hyperfluorescence of angioid streaks at the site of CNV.

The patient received monthly intravitreal ranibizumab $(0.5 \mathrm{mg} / 0.05 \mathrm{ml})$ injections on a pro re nata protocol for 2 months. The $\mathrm{CNV}$ was quiescent for 5 months. On his last visit date his best corrected visual acuity was 20/25 OD 20/30 OS.

Choroidal neovascularization $(\mathrm{CNV})$ is an important cause of vision loss in eyes with angioid streaks and occurs in approximately $42-84 \%$ of cases. While type 2 $\mathrm{NV}$ is the most common presenting anatomical subtype of NV to occur in the setting of angioid streaks, type 1 and mixed (type 1 and type 2) lesions occur in up to $16 \%$ of eyes [8]. Bruch's membrane (BrM) plays a critical role in retinal and choroidal physiology and regulates nutrient exchange and metabolic homeostasis [9]. BrM also serves an important mechanical function and separates the choroidal circulation from the outer retina. Interruptions in the mechanical integrity of BrM can result in dysregulation of regional growth factor gradients that together with a potential communication between retinal and choroidal compartments can culminate in CNV. Focal defects in BrM have been implicated in the pathophysiology of $\mathrm{CNV}$ in a range of conditions; however, this relationship is best exemplified in conditions with angioid streaks. The spatial relationship between Bruch's membrane and $\mathrm{CNV}$ can be precisely studied using OCTA where, as shown in this report, there is a close relationship between the morphology of BrM defects and the topographic pattern of CNV. The morphology of type $1 \mathrm{NV}$, with respect to the tangled morphology of pathological vessels, [10] shows many similarities to what has been described in AMD. This suggests that there is some
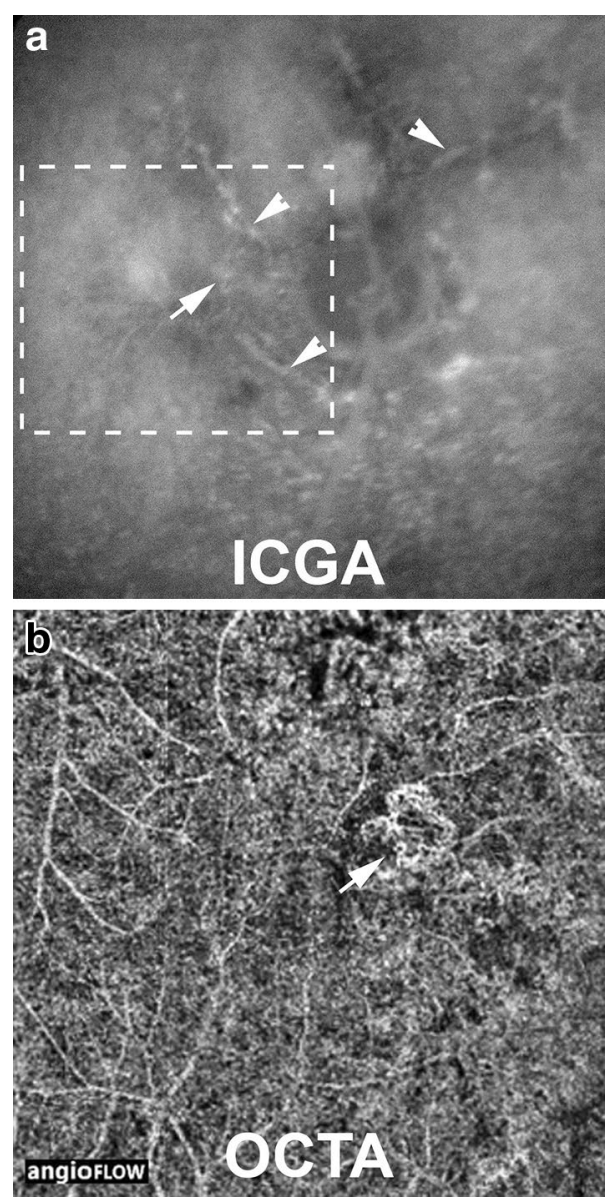

Fig. 3 Comparison between indocyanine green angiography and optical coherence tomographic angiography for visualizing type 1 neovascularization. Areas of type 1 NV (arrow) may be difficult to discern on ICGA (a) due to hyperfluorescent staining of angioid streaks (arrowheads) within areas of NV. In contrast, type 1 NV demonstrates a characteristic tangled morphology appearance on OCTA (b) that is easily appreciated. Inset on ICGA corresponds to the region of interest on OCTA image. Note the projection artifact of retinal vessels on the OCTA image.

overlap in biochemical stimuli that promote pathological angiogenesis in AMD and conditions associated with angioid streaks.

\section{Conclusions}

The multimodal imaging characteristics of angioid streaks have been previously reported [8, 11-13]. To our knowledge, this is the first report to demonstrate the morphology and spatial relationships of $\mathrm{CNV}$ and angioid streaks using OCTA. Our observations support the evidence that breaks in BrM are indeed the underlying pathology of AS [11]. The findings in this report implicate a critical role served by Bruch's membrane defects in the pathophysiology of CNV. In conclusion, 
multimodal imaging in combination with OCTA provides us with an excellent tool for the early detection and monitoring of AS related CNV. Those tools may enable improved visual outcomes for the management of $\mathrm{CNV}$ secondary to AS that often occurs in relatively young patient population with PXE.

\section{Consent}

Written informed consent was obtained from the patient for publication of this Case report and any accompanying images. A copy of the written consent is available for review by the Editor-in-Chief of this journal.

\begin{abstract}
Abbreviations
AS: angioid streaks; PXE: pseudoxanthoma elasticum; CNV: choroidal neovascularization; OCTA: optical coherence tomography angiography; BrM: Bruch's membrane; OD: oculus dexter (right eye); OS: oculus sinister (left eye); SD-OCT: spectral domain optical coherence tomography; RPE: retinal pigment epithelium; PDT: photodynamic therapy; Anti-VEGF: anti-vascular endothelial growth factor.
\end{abstract}

\section{Authors' contributions}

Participated in the sequence alignment and drafted the manuscript: $O G, C B$, KBF. Analysis of the images: OG, CB, KBF. All authors read and approved the final manuscript.

\section{Author details}

${ }^{1}$ LuEsther T. Mertz Retinal Research Center, Manhattan Eye, Ear and Throat Hospital, New York, NY, USA. ${ }^{2}$ Vitreous-Retina-Macula Consultants of New York, 460 Park Avenue, 5th Floor, New York, NY 10022, USA. ${ }^{3}$ Centre for Ophthalmology and Visual Sciences, Lions Eye Institute, University of Western Australia, Perth, Australia. ${ }^{4}$ Department of Ophthalmology, New York University School of Medicine, New York, NY, USA. ${ }^{5}$ Ophthalmology Department, Rabin Medical Center, Petach Tikva, Israel.

\section{Compliance with ethical guidelines}

\section{Competing interests}

K B. Freund is a consultant to Genentech, ThromboGenics, Ohr Pharmaceutical, Optos, Optovue, and Heidelberg Engineering (honorarium for each). The other authors have no conflicting interests to disclose.

\section{Financial support}

LuEsther T. Mertz Retinal Research Center, Manhattan Eye, Ear and Throat Hospital/North Shore Long Island Jewish Hospital, New York, NY, USA, and The Macula Foundation, Inc., New York, NY, USA. The funding organizations had no role in the design or conduct of this research.

Received: 26 June 2015 Accepted: 30 July 2015

Published online: 07 August 2015

\section{References}

1. Chassaing N, Martin L, Calvas P et al (2005) Pseudoxanthoma elasticum: a clinical, pathophysiological and genetic update including 11 novel ABCC6 mutations. J Med Genet 42:881-892. doi:10.1136/ jmg.2004.030171

2. Jensen OA (1977) Bruch's membrane in pseudoxanthoma elasticum Histochemical, ultrastructural, and x-ray microanalytical study of the membrane and angioid streak areas. Albrecht Von Graefes Arch Klin Exp Ophthalmol 203:311-320

3. Agarwal A, Patel P, Adkins T, Gass JDM (2005) Spectrum of pattern dystrophy in pseudoxanthoma elasticum. Arch Ophthalmol 123:923-928. doi:10.1001/archopht.123.7.923

4. Kirkwood BJ (2011) Angioid streaks. Insight J Am Soc Ophthalmic Regist Nurses. doi:10.1159/000300516

5. Brinkmann CK, Holz FG, Issa PC et al (2013) Treatment of choroidal neovascularization due to angioid streaks a comprehensive review. Retina 11:1214-1300. doi:10.1097/IAE.0b013e3182914d2b

6. Dansingani KK, Naysan J, Freund KB (2015) En face OCT angiography demonstrates flow in early type 3 neovascularization (retinal angiomatous proliferation). Eye 29:703-706

7. Freund KB, Engelbert M, Fine HF (2015) Individualizing the intravitreal anti-VEGF dosing regimen for long-term management of neovascular AMD. Ophthalmic Surg Lasers Imaging Retin 46:508-512. doi:10.3928/23258160-20150521-01

8. Nakagawa S, Yamashiro K, Tsujikawa A et al (2012) The time course changes of choroidal neovascularization in angioid streaks. Retina. doi:10.1097/IAE.0b013e31826b0bbe

9. Booij JC, Baas DC, Beisekeeva J et al (2010) The dynamic nature of Bruch's membrane. Prog Retin Eye Res 29:1-18. doi:10.1016/j. preteyeres.2009.08.003

10. Spaide RF (2015) Optical coherence tomography angiography signs of vascular abnormalization with antiangiogenic therapy for choroidal neovascularization. Am J Ophthalmol. doi:10.1016/j.ajo.2015.04.012

11. Issa PC, Finger RP, Holz FG, Scholl HPN (2009) Multimodal imaging including spectral domain OCT and confocal near infrared reflectance for characterization of outer retinal pathology in pseudoxanthoma elasticum. Investig Ophthalmol Vis Sci 50:5913-5918. doi:10.1167/iovs.09-3541

12. Zweifel SA, Imamura Y, Freund KB, Spaide RF (2011) Multimodal fundus imaging of pseudoxanthoma elasticum. Retina 31:482-491. doi:10.1097/ IAE.0b013e3181f056ce

13. Shiraki K, Kohno T, Moriwaki M, Yanagihara N (2001) Fundus autofluorescence in patients with pseudoxanthoma elasticum. Int Ophthalmol 24:243-248. doi:10.1023/A:1025433431654

\section{Submit your next manuscript to BioMed Central and take full advantage of:}

- Convenient online submission

- Thorough peer review

- No space constraints or color figure charges

- Immediate publication on acceptance

- Inclusion in PubMed, CAS, Scopus and Google Scholar

- Research which is freely available for redistribution

Submit your manuscript at www.biomedcentral.com/submit
( Biomed Central 